\title{
Dynamic traffic signal phase sequencing for an isolated intersection using ANFIS
}

\author{
Kingsley Monday Udofia ${ }^{1}$, Joy Omoavowere Emagbetere², Frederick Obataimen Edeko² \\ ${ }^{1}$ Department of Elect/Elect/Computer Engineering, University of Uyo, Uyo, Nigeria \\ ${ }^{2}$ Department of Electrical/Electronic Engineering, University of Benin, Benin City, Nigeria
}

Email address:

kingsleyudofia@uniuyo.edu.ng (K. M. Udofia),miracle5ng@yahoo.com (J. O. Emagbetere), frededeko@yaho.co.uk (F. O. Edeko)

\section{To cite this article:}

Kingsley Monday Udofia, Joy Omoavowere Emagbetere, Frederick Obataimen Edeko. Dynamic Traffic Signal Phase Sequencing for an Isolated Intersection Using ANFIS. Automation, Control and Intelligent Systems. Vol. 2, No. 2, 2014, pp. 21-26.

doi: 10.11648/j.acis.20140202.12

\begin{abstract}
This paper presents a traffic signal phase sequencing using adaptive neuro-fuzzy inference system (ANFIS) technique. The system is designed to emulate traffic expert on the selection of the appropriate phase to be given right-ofway at an isolated intersection based on the prevailing traffic situation. Inputs (queuelength and waiting time of vehicles) from traffic detectors are used to determine the selection of the next green phase. We evaluated the developed model for five different common traffic scenarios using MATLAB. The results obtained indicates that the developed model adaptively and effectively selects a phase to be given next green signal after considering the traffic situation and the nature of the intersection in question.
\end{abstract}

Keywords: Adaptive, Neuro-Fuzzy Inference System, Phase Sequencing, Vehicle Traffic Control, Isolated Intersection

\section{Introduction}

The use of traffic signals is considered to be one of the most effective ways to control traffic at intersections [1]. Traffic signals are used to assign the right-of-way to intersecting traffic streams for the purpose of ensuring that all streams are served safely and without excessive delay.

The working of the traffic signal control currently deployed in many intersections is based on predetermined and fixed signal phase sequencing [2]. The streams constituting each phase and the order in which the corresponding phases come on are fixed. The right of way are always given to the phases in a fixed sequence irrespective of whether there is traffic in a phase or not, thus leading to unnecessary delays of traffic at the intersection [2]. This has instigated various ideas and scenarios by traffic engineers to solve the traffic problem [3 - 7]. To design an intelligent and efficient traffic control system, a number of parameters that represent the status of the traffic conditions must be identified and taken into consideration [8].

In this paper, we adopted adaptive neuro-fuzzy inference system (ANFIS) to model a real time, adaptive traffic signal phase control for an isolated intersection. ANFIS uses a hybrid training method to automatically generate fuzzy rules according to a given input-output datasets. The proposed case study is a real four-way intersection, located in the urban area of Uyo (Nigeria), with severe traffic congestion.

\section{ANFIS Architecture}

ANFIS works by applying neural learning rules to identify and tune the parameters and structure of a Fuzzy Inference System (FIS). A typical architecture of an ANFIS, in which a circle indicates a fixed node, whereas a square indicates an adaptive node, is shown in figure 1. For simplicity, we assume that the examined FIS has two inputs and one output. For a first-order Sugeno fuzzy model, a typical rule set with two fuzzy "if then" rules can be expressed as follows:

Rule 1: If $x$ is $\mathrm{A}_{1}$ and $y$ is $\mathrm{B}_{1}$, then $z_{1}=f_{1}(x)$

$$
=p_{1} x_{1}+q_{1} x_{2}+r_{1}
$$

Rule 2: If $x$ is $\mathrm{A}_{2}$ and $y$ is $\mathrm{B}_{2}$, then $\mathrm{z}_{2}=f_{2}(x)$

$$
=p_{2} x_{1}+q_{2} x_{2}+r_{2}
$$

Where $\mathrm{x}$ and $\mathrm{y}$ are the two crisp inputs, and Ai and Bi are the linguistic labels associated with the node function.

As indicated in figure 1, the system has a total of five 
layers. The functioning of each layer is described as follows [9].

Input node (Layer 1): Nodes in this layer contains membership functions. Parameters in this layer are referred to as premise parameters. Every node $\mathrm{i}$ in this layer is a square and adaptive node with a node function:

$$
O_{i}^{1}=\mu_{A_{i}}(x) \text { for } i=1,2
$$

Where $\mathrm{x}$ is the input to node $\mathrm{i}$, and $\mathrm{Ai}$ is the linguistic label (short, long, etc.) associated with this node function. In other words, $O_{i}^{1}$ is the membership function of $\mathrm{Ai}$ and it specifies the degree to which the given $\mathrm{x}$ satisfies the quantifier Ai.

Rule nodes (Layer 2): Every node in this layer is a circle node labeled $\prod$, whose output represents a firing strength of a rule. This layer chooses the minimum value of two input weights. In this layer, the AND/OR operator is applied to get one output that represents the results of the antecedent for a fuzzy rule, that is, firing strength. It means the degrees by which the antecedent part of the rule is satisfied and it indicates the shape of the output function for that rule. The node generates the output (firing strength) by cross multiplying all the incoming signals:

$$
O_{i}^{2}=\mu_{A_{i}}(x) \times \mu_{B_{i}}(x) \text { for } i=1,2
$$

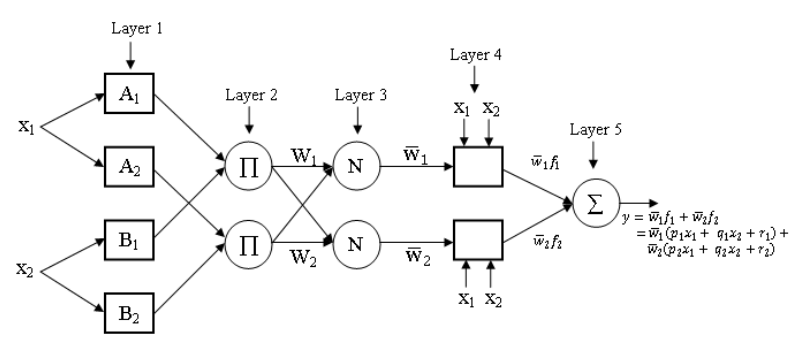

Figure 1. ANFIS architecture.

Average nodes (Layer 3): Every node in this layer is a circle node labeled $\mathrm{N}$. The ith node calculates the ratio between the ith rule's firing strength to the sum of all rules' firing strengths. Every node of these layers calculates the weight, which is normalized. For convenience, outputs of this layer are called normalized firing strengths.

$$
\bar{w}_{i}=\frac{w_{i}}{w_{1}+w_{2}}, \quad \text { for } i=1,2
$$

Consequent nodes (Layer 4): This layer includes linear functions, which are functions of the input signals. This means that the contribution of ith rule towards the total output or the model output and/or the function defined is calculated. Every node $\mathrm{i}$ in this layer is a square node with a node function:

$$
O_{i}^{4}=\bar{w}_{i} f_{i}=\bar{w}_{i}\left(p_{i} x_{1}+q_{i} x_{2}+r_{i}\right)
$$

Where wi is the output of layer 3, and \{pi, qi, ri $\}$ is the parameter set of this node. These parameters are referred to as consequent parameters.
Output node (Layer 5): The single node in this layer is a fixed node labeled $\Sigma$, which computes the overall output by summing all incoming signals:

$$
O_{i}^{5}=\text { overalloutput }=\sum_{i} \bar{w}_{i} f_{i}=\frac{\sum_{i} w_{i} f_{i}}{\sum_{i} w_{i}}
$$

The tuning or training procedure for ANFIS is achieved based on the batch learning technique using input-output training dataset. During training ANFIS optimizes the adjustable parameters by comparing ANFIS output with trained data. Each period of training is divided into two phases. In the first phase, the consequent parameters are adjusted with Least-squares method and in the second phase, the premise parameters are adjusted with gradient descent (back propagation) method.

\section{Methodology}

In this paper, we adopted adaptive neuro-fuzzy inference system (ANFIS) to model a real time, adaptive traffic signal phase control for an isolated intersection. ANFIS uses a hybrid training method to automatically generate fuzzy rules according to a given input-output datasets.

In the design, the following requirements were taken into consideration:

- to reduce the delay time of waiting vehicles;

- to avoid traffic congestion (queue lengths); and

- to avoid conflicts at the intersection.

There are two major ways of controlling phase sequence in order to avoid conflicts at traffic intersections, namely, fixed and variable phase sequences. In fixed phase sequence, the sequencing of the phases are pre-determined and fixed despite the prevailing traffic conditions at the intersection. Whereas, the sequencing in a variable phase sequence is determined by the existing traffic situations. The variable phase sequence has the flexibility to fully adapt to the traffic flow fluctuations.

In this work, a variable phase sequence control method was adopted. This method uses queuelengths and waiting time as input parameters. The decision output is to select a phase with worst traffic condition in an intersection.

In order to effectively control traffic at an intersection adaptively, the ANFIS model used two sets of input parameters, namely, the queuelengths $(\mathrm{q})$, and waiting time of each phase (wt) in an intersection. These inputs parameters are obtained for each phase using vehicle detectors. Figure 2 shows the architecture of the proposed ANFIS-based traffic signal phase controller.

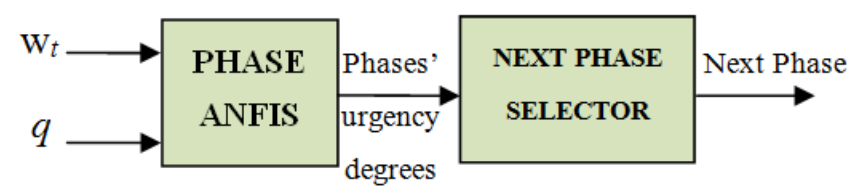

Figure 2. Block diagram of the proposed ANFIS-based traffic signal phase controller. 
The queuelength $(q)$ indicates the number of vehicles in a phase during a red light phase, and is given as the sum of the residue vehicles since the last green signal and the arrival during the current red signal as given by equation (6).

$$
q=q_{g r}+\sum V_{r}^{i}
$$

where $q_{g r}$ is the number of vehicles that did not exit the intersection in the green phase and $\sum V_{r}^{i}$ is the sum of arrived vehicles in the $i^{\text {th }}$ second for the red phase. In general queuelength depends on the number of vehicles arriving and leaving an approach at a given time interval as given by equation (7) [15].

$$
q_{n}(t+1)=q_{n}(t)-D_{n}(t)+A_{n}(t)
$$

where $q_{n}(t+1)$ is the number of queued vehicles at time t, $q_{n}(t)$ is the residual queue from previous periods, and $A_{n}(t)$ and $D_{n}(t)$ are the vehicles arrivals and departures within time interval $[\mathrm{t},(\mathrm{t}+1)]$.

The waiting time $(\mathrm{Wt})$ indicates the duration of time, vehicles have waited in the queue since the elapse of the last green phase signal and is computed using equation (8). This input parameter is considered so as to avoid the vehicles waiting too long for the green signal.

$$
W_{t}= \begin{cases}t_{v 1} & \text { if } q_{0}=0 \\ t_{R} & \text { if } q_{0}>0\end{cases}
$$

where $t_{v 1}$ is the time between the arrival of the first vehicle in a queue during the current red phase and the next green phase signal, $t_{R}$ is the current red phase time measured from the end of the last green phase signal, and $q_{0}$ is the number of vehicles in the queue at the end of the last green phase signal.

The phase ANFIS was designed to use waiting time and queuelength to determine the urgency degree $\left(\operatorname{Urg}_{P h}\right)$ separately for each phase. To adaptively and effectively determine the phase in an intersection to be given the right of way at a particular time taking cognizance the prevailing traffic situation, certain considerations were made. It is expected for a phase with long queuelength to have high probability of having the next green signal. In cases where there are vehicles, though few, which have stayed in another phase for a period of time longer than necessary, the decision to chose the phase for the next green signal becomes complex. To determine the optimum solution to this situation the urgency degree of each phase $\left(U r g_{P h}\right)$ which combines the individual worsening effect of these two factors must computed as given in equation (9).

$$
\operatorname{Urg}_{P h}=W_{e}+Q_{e}
$$

where $W_{e}$ is the effect of waiting time, and $Q_{e}$ is the effect of phase queuelength.

The effect of waiting time $W_{e}$ was considered in the design such that the same actual waiting time $w_{t}$ but different maximum waiting time $w_{t(\max )}$, cannot have the same worsening effect; the one with lower $w_{t(\max )}$ should have higher effect as expressed as in equation (10).

$$
W_{e}=\frac{w_{t}}{w_{t(\max )}}
$$

The worsening effect of waiting time $W_{e}$ is additive. Hence, equation (10) can be rewritten as equation (11).

$$
W_{e}=+\frac{w_{t}}{w_{t(\max )}}
$$

The effect of queuelength was measured by equation (12), where $Q_{e}$ is the worsening effect of queuelength, $q$ is the actual queuelength and $q_{(\max )}$ is the phase's maximum queuelength capacity. Two phases with the same $q$ but different $q_{(\max )}$ cannot have the same worsening effect; the one with lower $q_{(\max )}$ have higher effect.

$$
Q_{e}=\frac{q}{q_{(\max )}}
$$

Thus, the worsening effect of queuelength $Q_{e}$ is additive. Hence, equation (12) was rewritten as;

$$
Q_{e}=+\frac{q}{q_{(\max )}}
$$

Substituting equations (11), (13) into equation (9), we have an expression for the overall effects of traffic factors affecting a phase at a time, called urgency degree $\left(U r g_{P h}\right)$, as given in equation (14).

$$
\operatorname{Urg}_{P h}=\frac{w_{t}}{w_{t(\max )}}+\frac{q}{q_{(\max )}}
$$

Since a phase with no vehicle in queue does not need green signal, for equation (14) to be successfully adapted in traffic control, the phase urgency degree $U r g_{P h}$, must be zero (0) whenever the phase's queuelength $q$, is zero (0). Thus, equation (14) is rewritten as in equation (15).

$$
\operatorname{Urg}_{P h}= \begin{cases}{\left[\frac{w_{t}}{w_{t(\max )}}+\frac{q}{q_{(\max )}}\right],} & \text { for } q>0 \\ 0, & \text { for } q=0\end{cases}
$$

$w_{t(\max )}$ and $q_{(\max )}$ can be determine based on the prevailing factors of the intersection's traffic such as traffic density and lane length.

Equation (15) is used to construct the input/output datasets for the training the ANFIS models as described in section 4 .

The Next Phase Selector module uses urgency degrees of all phases in the intersection to determine the phase to be given the next green signal. It selects the phase with the maximum urgency degree the intersection (16).

$$
U r g_{N x t P h}=\max _{1 \leq i \leq n}\left\{U r g_{P h}(i)\right\}
$$

where $U r g_{N x t P h}$ is the urgency degree of the Next phase, $\operatorname{Urg}_{P h}(i)$ is the urgency degree of individual phases constituting the intersection, and $\mathrm{n}$ is the number of the phases considered. 


\section{ANFIS Models Designing}

It is our target to develop a traffic control model that will automatically and adaptively take traffic decisions just like expert/professional traffic wardens. Although ANFIS model could achieve this, there is need to develop and train the ANFIS with datasets that reflect expert warden's judgments at different traffic situations.

The input-output training dataset for each phase ANFIS model was developed from parameters obtained from a case study intersection located in Uyo, Nigeria using equation (15). The intersection schematic is shown in figure 3 with four phases labeled A, B, C and D. Phases A and C are double lanes while phases $\mathrm{B}$ and $\mathrm{D}$ are single lane. To develop the dataset used in training each phase ANFIS:

- $\quad$ the range of waiting time, $w_{t}$, was $0-500$ seconds;

- $\quad$ the range of queuelength, $q$, was $0-300$ vehicles;

- $\quad$ the threshold waiting time, $w_{t(\max )}$, was two minutes (120 seconds); and

- $\quad$ the threshold queuelength, $q_{(\max )}$, was 34 vehicles for double-lane phases (A and $\mathrm{C}$ ) and 17 vehicles for single-lane phase (B and D).

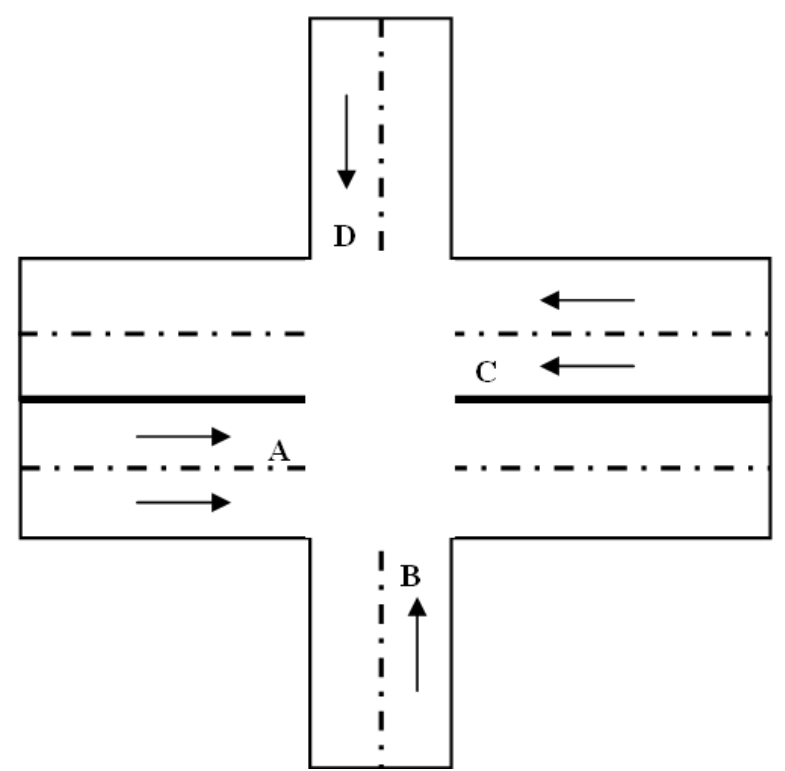

Figure 3. The schematic diagram of the case study intersection.

In designing the ANFIS we used MATLAB Anfis editor. Each phase ANFIS model passed through the following four steps:

- Load data;

- $\quad$ Generate fuzzy inference system (FIS);

- Train FIS; and

- $\quad$ Test FIS model.

The ANFIS model used Grid Partition method for FIS generation. Grid partition divides the data space into rectangular subspaces using axis-paralleled partition based on pre-defined number of membership functions and their types in each dimension. The generated FIS includes two inputs and one output. The input variables are: waiting time $\left(w_{t}\right)$ and queuelength $(q)$. The type of membership function used is the generalized bell because of its smoothness and non-linearization ability coupled with the degree of freedom to adjust the steepness at the crossover points. The number of membership functions for waiting time $\left(w_{t}\right)$ and queuelength $(q)$ is six each. The output field is the urgency degree. Membership function type of output variable is linear. Figures 4 and 5 show tuned membership functions of each input in the ANFIS model. The structure of the tuned FIS is shown in figure 6 and contains 36 rules.

The ANFIS model used hybrid optimization method (least-squares error and back propagation gradient descent methods) to train the membership function parameters to emulate the training data.

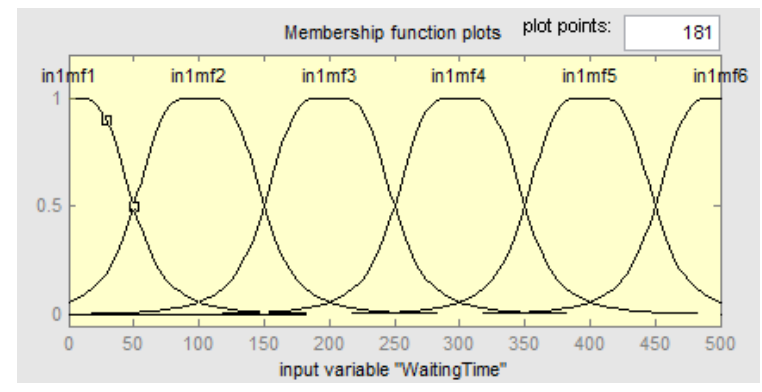

Figure 4. Tuned membership functions for waiting time.

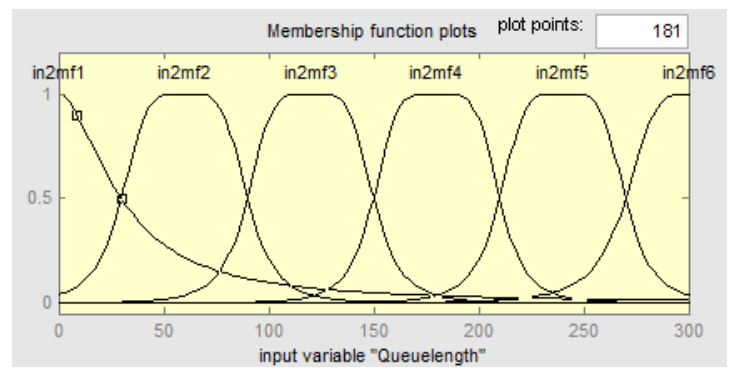

Figure 5. Tuned membership functions for queuelength

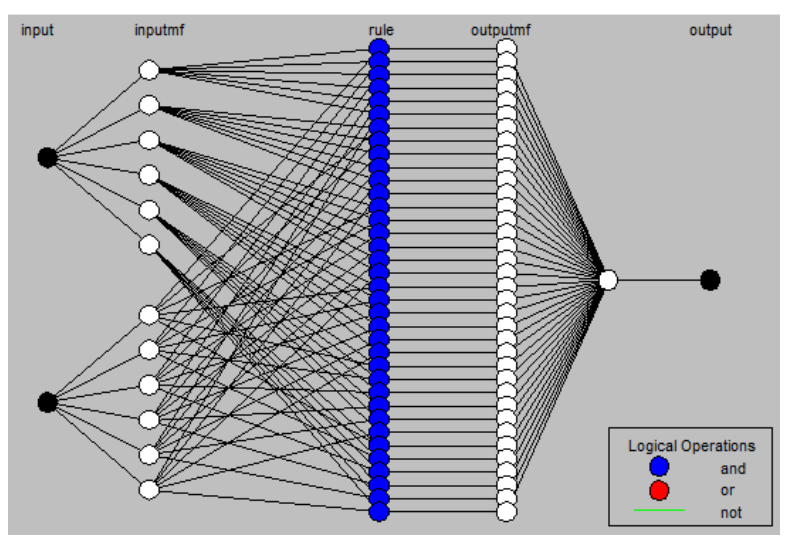

Figure 6. Structure of the Phase ANFIS model.

A block diagram of the trained phase ANFIS model is shown in figure 7 . It can be seen that the model has two input variables (waiting time and queuelength) and an output variable (urgency degree). The input-output views of the ANFIS model in figures 8 and 9 illustrate how the Urgency degree will respond to varying values of waiting time and queuelength. 


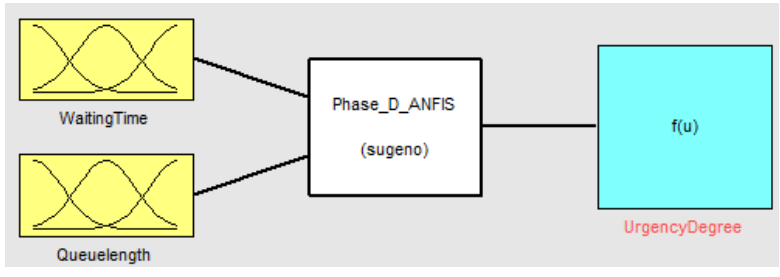

Figure 7. Structure of the trained Phase ANFIS model.

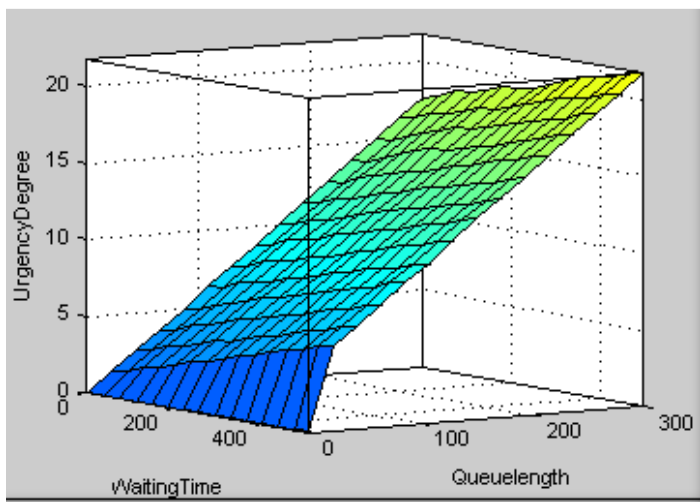

Figure 8. $3 D$ plot of waiting time and queuelength (inputs variables) VS Urgency degree (output variable).

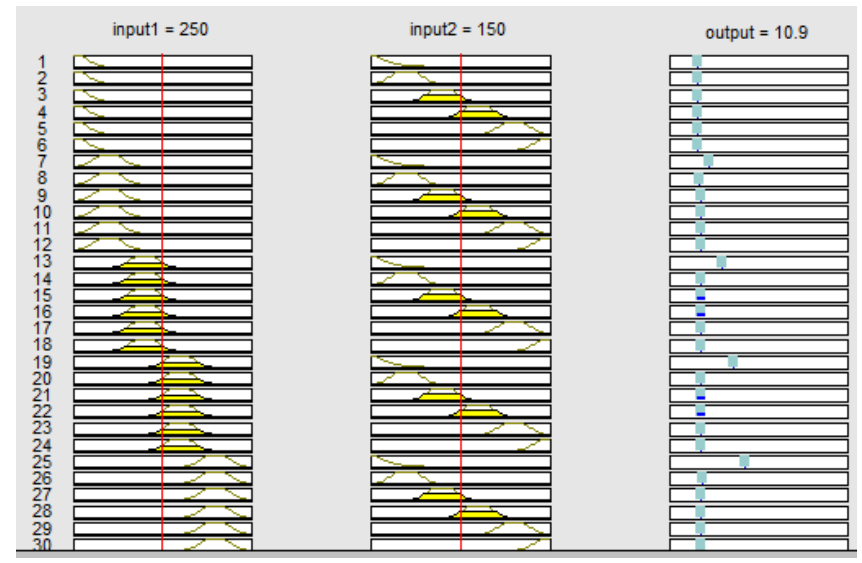

Figure 9. MATLAB rule viewer showing fuzzy rules and membership functions.

\section{Result and Discussion}

We perform five different evaluations of the ANFIS models based on real traffic scenarios. In each evaluation, we vary the inputs (waiting time and/or queuelength) of a phase(s) and we observed the effect in the choice of the next phase. The MATLAB Anfis rule viewer of each of the phases was used to extract the urgency degree for each set of input variables.

CASE 1: A phase with a high queuelength compared to other phases

In this evaluation, the effect of having a high queue in a phase compared to the other phases of the intersection, in the selection of the next green phase is considered. Assuming that phase $\mathrm{C}$ has queuelength of 100 vehicles which is high compared to other phases as can be seen in table 2 .
Table 2. Effect of Congested phase on next phase selection.

\begin{tabular}{ccccc}
\hline $\begin{array}{c}\text { ANFIS } \\
\text { Model }\end{array}$ & $\begin{array}{c}\text { Waiting } \\
\text { Time (s) }\end{array}$ & $\begin{array}{c}\text { Queuelength } \\
\text { (veh) }\end{array}$ & $\begin{array}{c}\text { Urgency } \\
\text { Degree }\end{array}$ & $\begin{array}{c}\text { Selected } \\
\text { Phase }\end{array}$ \\
\hline Phase A & 100 & 30 & 1.79 & \\
Phase B & 100 & 20 & 2.01 & Phase C \\
Phase C & 100 & 100 & 3.77 & \\
Phase D & 100 & 25 & 2.38 & \\
\hline
\end{tabular}

As can be observed from table 2, phase $\mathrm{C}$ which has the highest urgency degree of 3.77 is selected as the next phase to be given green signal. This signifies that the phase that is most congested among all phases in an intersection will be preferred for the next green phase.

CASE 2: A single-lane phase and a double-lane phase with high queuelength of same magnitude

In this evaluation, a situation where there are high queue of the same volume of vehicles in a single-lane phase and a double-lane phase is considered. Assuming that phase A (double lanes) and phase B (single lane) have a queuelength of 100 vehicles each as in table 3 .

Table 3. Effect of number of lanes in a route on next phase selection.

\begin{tabular}{ccccc}
\hline $\begin{array}{r}\text { ANFIS } \\
\text { Model }\end{array}$ & $\begin{array}{c}\text { Waiting } \\
\text { Time (s) }\end{array}$ & $\begin{array}{c}\text { Queuelength } \\
\text { (veh) }\end{array}$ & $\begin{array}{c}\text { Urgency } \\
\text { Degree }\end{array}$ & $\begin{array}{c}\text { Selected } \\
\text { Phase }\end{array}$ \\
\hline Phase A & 100 & 100 & 3.77 & \\
Phase B & 100 & 100 & 6.72 & Phase B \\
Phase C & 100 & 40 & 2.01 & \\
Phase D & 100 & 25 & 2.30 & \\
\hline
\end{tabular}

As can be observed from table 3 , despite the fact that both phases $\mathrm{A}$ and $\mathrm{B}$ have the same waiting times and queuelengths, phase $\mathrm{B}$ which is a single-lane route has a higher urgency degree of 6.72 and thus, is selected as the next phase to be given green signal. This is because the same number of vehicles in a single-lane route will occupy double the distance of that of a double-lane route; thus a single-lane route will be said to be experiencing more congestion.

CASE 3: No vehicle in a phase

In this evaluation, a case where there is no vehicle waiting in a particular phase is considered. Assuming that there is no vehicle in phase $\mathrm{D}$ as given in table 4 .

Table 4. Effect of having no vehicle in a phase on next phase selection.

\begin{tabular}{ccccc}
\hline $\begin{array}{c}\text { ANFIS } \\
\text { Model }\end{array}$ & $\begin{array}{c}\text { Waiting } \\
\text { Time (s) }\end{array}$ & $\begin{array}{c}\text { Queuelength } \\
\text { (veh) }\end{array}$ & $\begin{array}{c}\text { Urgency } \\
\text { Degree }\end{array}$ & $\begin{array}{c}\text { Selected } \\
\text { Phase }\end{array}$ \\
\hline Phase A & 100 & 30 & 1.79 & \\
Phase B & 100 & 21 & 2.09 & Phase B \\
Phase C & 100 & 40 & 2.01 & \\
Phase D & 100 & 0 & 0 & \\
\hline
\end{tabular}

As can be observed from table 4, phase $\mathrm{D}$ with no vehicle has an urgency degree of 0 and hence, can never be selected as the next phase to be given green signal. Whenever there is no vehicle waiting in a route, it will be useless assigning green signal to that phase.

CASE 4: A Phase with low queuelength and very high waiting time

In this evaluation, the effect of a phase with low queuelength and high waiting time in the selection of the 
next green phase is considered. Assuming that phase $\mathrm{C}$ has 5 vehicles with a waiting time of 400 seconds as given in table 5 .

Table 5. Effect of a phase with low volume of vehicles and very high waiting time on next phase selection.

\begin{tabular}{ccccc}
\hline $\begin{array}{c}\text { ANFIS } \\
\text { Model }\end{array}$ & $\begin{array}{c}\text { Waiting } \\
\text { Time (s) }\end{array}$ & $\begin{array}{c}\text { Queuelength } \\
\text { (veh) }\end{array}$ & $\begin{array}{c}\text { Urgency } \\
\text { Degree }\end{array}$ & $\begin{array}{c}\text { Selected } \\
\text { Phase }\end{array}$ \\
\hline Phase A & 100 & 60 & 2.60 & \\
Phase B & 100 & 20 & 2.01 & Phase C \\
Phase C & 400 & 5 & 3.41 & \\
Phase D & 100 & 30 & 2.65 & \\
\hline
\end{tabular}

As can be observed from table 5 , though phase $\mathrm{C}$ has a very low queuelength, its urgency degree is the highest because of the very high waiting time of vehicles and hence, is selected as the next phase to be given green signal. Although a phase has few vehicles compared to other phases, it should not be denied a right of way most especially having waited for a long time.

CASE 5: The current green signal phase has a very high queuelength compared to other phases

In this evaluation, a case where the phase currently being served green signal has a very high queuelength compared to other phases in an intersection is considered. Assuming that phase $\mathrm{A}$ is the current green phase with queuelength of 200 vehicles and zero (0) waiting time.

Table 6. Effect of Congested phase on next phase selection.

\begin{tabular}{ccccc}
\hline $\begin{array}{c}\text { ANFIS } \\
\text { Model }\end{array}$ & $\begin{array}{c}\text { Waiting } \\
\text { Time (s) }\end{array}$ & $\begin{array}{c}\text { Queuelength } \\
\text { (veh) }\end{array}$ & $\begin{array}{c}\text { Urgency } \\
\text { Degree }\end{array}$ & $\begin{array}{c}\text { Selected } \\
\text { Phase }\end{array}$ \\
\hline Phase A & 0 & 200 & 5.88 & \\
Phase B & 100 & 21 & 2.09 & \multirow{2}{*}{ Phase A } \\
Phase C & 100 & 40 & 2.01 & \\
Phase D & 100 & 25 & 2.30 & \\
\hline
\end{tabular}

As can be observed from table 6, though phase $\mathrm{A}$ is the current green phase, it has the highest urgency degree because of the congestion level compared to other phases and hence, its green signal is extended. If the phase currently being served green signal is congested compared to the other phase, it is wisdom to extend the green signal.

\section{Conclusion}

The conventional traffic control system uses a fixed phase sequence where the order of corresponding phases is fixed irrespective of the prevailing traffic conditions at an intersection. Thus, the tendency of assigning green signal to a phase with no vehicle or an over-congested phase, irrespective of the fact that other phases have low traffic. These are issues that do not promote economic growth in any society as man-power hours are lost, hence productivity is greatly affected, aside the personal discomforts experienced by the motorists.

In this paper, we developed a model that adaptively and effectively control phase sequencing at traffic isolated intersection using ANFIS. A mathematical model for the design of input-output datasets used in the training and tuning of each phase ANFIS model was also developed. We evaluated the developed model for five different common traffic scenarios using MATLAB. The results obtained indicated that the developed model effectively selects a phase to be given next green signal after considering the traffic situation and the nature of the intersection in question.

\section{References}

[1] E.A. Mueller. Aspects of the history of traffic signals. IEEE Transactions on Vehicular Technology, 19(1):6 -17, February 1970.

[2] G.E.M.D.C. Bandara et at.: Application of fuzzy logic in intelligent traffic control systems, National University of Singapore, CIRAS, 2003

[3] M. R. A. Purnomo et al.: Development of a low cost smart traffic controller system, European Journal of Scientific Research, 2009, 32(4): 490 - 499

[4] Niittymaki J. et al.: Fuzzy Traffic Signal Control and a New Interface Method - Maximal Fuzzy Similarity. In: Proc., The 13th Mini-EURO Conf. (Handling Uncertainty in the Analysis of Traffic and Transportation Systems) and the 9th Mtg. EURO Working Group on Transportation Intermodality, Sustainability and Intelligent Transportation Systems, Bari, Italy, 2002, 716-728.

[5] Zhang L, Li H, Prevedouros P D. Signal control for oversaturated intersections using fuzzy logic. In: Proc. of 84th Transp. Res. Bd. Ann. Mtg., Washington, D.C., 2005

[6] Nakatsuyama M, Nagahashi H, Nishizuka N. Fuzzy logic phase controller for traffic junctions in the one-way arterial road. In: Proc., IFAC 9th Triennial World Cong., Budapest, Hungary, 1984, 2865-2870

[7] Tan, K.K., Khalid, M., Yusof, R.: Intelligent Traffic Lights Control by Fuzzy Logic. Malaysian Journal of Computer Science 9(2), 29-35 (1996)

[8] Pappis C. and Mamdani E.: A fuzzy logic controller for a traffic junction, IEEE Trans. Systems, Man, and Cybernetics SMC-7, 1977, 7(10): 707-717.

[9] J.S.R. Jang: ANFIS: Adaptive-network-based Fuzzy Inference Systems. IEEE Trans, Syst, Man Cybern., 23(3), pp. 665-685, 1993.

[10] Wenteng M.: A Real-time Performance Measurement System for Arterial Traffic Signals. A Ph.D thesis, Graduate School, University of Minnesota, 2008. 